\title{
Respiratory Syncytial Virus Infection Rates with Limited Use of Palivizumab for Infants Born at 29 to 31+6/7 Weeks Gestational Age
}

\author{
Brandi Newby and Todd Sorokan
}

\begin{abstract}
Background: Immunoprophylaxis with palivizumab can reduce respiratory syncytial virus (RSV) infections and hospitalizations. Criteria in British Columbia limit the use of palivizumab to infants born at 29 to $31+6 / 7$ weeks gestational age, which differ from guidelines of the American Academy of Pediatrics (AAP) and the Canadian Paediatric Society (CPS).

Objective: To determine whether the limited use of palivizumab affected the frequency of hospital visits by RSV-positive infants (termed "RSV-positive hospital visits") who received approval for palivizumab and those who met the AAP/CPS criteria but did not receive approval for palivizumab.

Methods: Data sets were generated for the period May 1, 2008, to April 30,2011 , to identify infants with gestational age of 29 to $31+6 / 7$ weeks who were born in or transferred to the Fraser Health authority, RSVpositive results for infants less than 12 months of age who had visited Fraser Health sites and BC Children's Hospital, and palivizumab approvals. Infants were matched across these 3 data sets through their personal health numbers.
\end{abstract}

Results: The study included 359 infants born at 29 to $31+6 / 7$ weeks, of whom 297 met the AAP/CPS criteria. However, only 46 of these 297 received approval for palivizumab according to the BC criteria. Sixteen (4.5\%) of the 359 infants had RSV-positive hospital visits during the RSV season (November through March). Of the 46 infants who received approval for palivizumab, 2 (4.3\%) had RSV-positive hospital visits, and of the 251 who met the AAP/CPS criteria but did not receive palivizumab approval, 14 (5.6\%) had RSV-positive hospital visits. Of the 359 infants, $6(1.7 \%)$ had RSV-positive results while admitted to the neonatal intensive care unit, and $10(2.8 \%)$ tested positive for RSV during a subsequent hospital visit.

Conclusions: The frequency of RSV-positive hospital visits did not differ between infants who received and those who did not receive approval for palivizumab in the Fraser Health authority. Limited use of palivizumab for RSV prophylaxis led to reasonable rates of RSV-positive hospital visits in the study population.

Keywords: respiratory syncytial viruses; infant, premature; antibodies, monoclonal, humanized

\section{RÉSUMÉ}

Contexte : L'immunoprophylaxie à l'aide du palivizumab peut permettre de réduire le nombre de cas d'infection par le virus respiratoire syncytial (VRS) et d'hospitalisation qui en résulte. Les critères de la ColombieBritannique restreignent l'utilisation du palivizumab aux nourrissons dont l'âge gestationnel se situait entre 29 semaines et 31 semaines et 6 jours. En cela, ils different des lignes directrices de l'American Academy of Pediatrics (AAP) et de la Société canadienne de pédiatrie (SCP).

Objectif : Vérifier si l'utilisation restreinte du palivizumab a eu un effet sur la fréquence des visites à l'hôpital de nourrissons séropositifs pour le VRS pour lesquels on a autorisé la prescription de palivizumab et de ceux qui ont satisfait aux critères de l'AAP et de la SCP, mais pour lesquels la prescription de palivizumab n'était pas autorisée.

Méthodes : Des ensembles de données ont été produits pour la période s'étendant du 1er mai 2008 au 30 avril 2011 afin d'identifier : les nourrissons nés dans les établissements de la Régie de la santé du Fraser ou y ayant été transférés dont l'âge gestationnel se situait entre 29 semaines et 31 semaines et 6 jours; les résultats positifs pour le VRS chez les nourrissons de moins de 12 mois ayant visité les établissements de la Régie de la santé du Fraser et l'Hôpital pour enfants de la ColombieBritannique; et les cas où l'on a autorisé la prescription de palivizumab. On a assorti les données des nourrissons entre les trois ensembles à l'aide de leur numéro d'assurance-maladie personnel.

Résultats : L'étude a recensé 359 nourrissons dont l'âge gestationnel se situait entre 29 semaines et 31 semaines et 6 jours. Parmi eux, 297 répondaient aux critères de l'AAP et de la SCP. Or, le traitement par palivizumab n'a été accordé qu’à 46 de ces 297 nourrissons selon les critères de la Colombie-Britannique. Seize (4,5\%) des 359 nourrissons qui avaient visité l'hôpital au cours de la saison du VRS (novembre à mars) se sont révélés séropositifs pour le VRS. Parmi les 46 nourrissons admissibles au traitement par palivizumab, deux (4,3\%) ont visité l'hôpital et se sont avérés séropositifs pour le VRS. Parmi les 251 autres enfants répondant aux critères de l'AAP et de la SCP, mais n'ayant pas été autorisés à recevoir du palivizumab, 14 (5,6 \%) ont visité l'hôpital et se sont révélés séropositifs pour le VRS. Parmi les 359 nourrissons, 6 (1,7\%) se sont avérés séropositifs pour le VRS alors qu'ils étaient admis à l'unité de soins intensifs néonatals et 10 (2,8\%) ont été déclarés séropositifs pour le VRS lors d'une visite subséquente à l'hôpital. 


\section{Can J Hosp Pharm. 2017;70(1):13-8}

Conclusions : La fréquence des visites de nourrissons séropositifs pour le VRS aux établissements de la Régie de la santé du Fraser n'était pas différente entre ceux qui étaient autorisés à recevoir du palivizumab et ceux qui n’y étaient pas autorisés. Une utilisation restreinte du palivizumab pour la prophylaxie du VRS a donné des taux raisonnables de visites à l'hôpital par les nourrissons séropositifs pour le VRS dans la population de l'étude.

Mots clés : virus respiratoire syncytial; nourrisson, prématuré; anticorps, monoclonaux, humanisés

\section{INTRODUCTION}

$\mathrm{R}$ espiratory syncytial virus (RSV) is the most common cause of lower respiratory tract infection in infants and young children. Those at greatest risk for severe RSV infection include premature infants in the first 6 months of life, children with underlying cardiac or pulmonary disease in the first 2 years of life, and children who are immunocompromised. RSV infection can be reduced by simple measures such as handwashing and avoidance of infected individuals. A monoclonal antibody against RSV, palivizumab, is available in many countries. The IMpact-RSV Study Group reported a 55\% reduction in RSV-related hospitalization with palivizumab prophylaxis for infants at high risk for severe RSV infection. ${ }^{1}$ Given the cost and logistics of administering palivizumab, several organizations around the world have developed guidelines for its use. ${ }^{2-6}$

In British Columbia, the BC RSV Immunoprophylaxis Task Force included neonatologists, cardiologists, oncologists, nurses, and pharmacists. The task force established the criteria defining which infants should receive RSV prophylaxis with palivizumab in the province for each RSV season. The authors of the current article represented their health region on the BC RSV task force but were not involved in selecting the criteria for RSV prophylaxis. The BC criteria have differed from guidelines of the American Academy of Pediatrics (AAP) and the Canadian Paediatric Society (CPS) for several years. During the period 2008 to 2011, the most significant difference involved restrictions on the use of palivizumab for infants born at 29 to $31+6 / 7$ weeks gestational age. According to the BC criteria, prophylaxis among these infants was limited to those born after September 1 and having 3 or more of the following risk factors: male sex, birth weight less than or equal to the 10th percentile, daycare attendance, sibling less than 5 years of age living in the same home, 5 or more people living in the household, and 2 or more smokers in the household. These risk factors were adapted from the validated risk scoring tool for RSV prophylaxis in infants born at 33 to 35 weeks gestational age. ${ }^{7}$ It is our understanding that the $\mathrm{BC}$ criteria were selected after review of guidelines from other countries, with the goal of providing palivizumab to the highest-risk infants, to minimize RSV-related hospitalizations, and also to ensure program sustainability. In contrast to the $\mathrm{BC}$ criteria, the AAP/CPS guidelines in effect over the period 2008 to 2011 recommended palivizumab prophylaxis for infants born at 29 to $31+6 / 7$ weeks gestational age if their age was less than 6 months at the start of the RSV season (i.e., born after May 1), with no requirement for additional risk factors. ${ }^{2,3}$ Because the $\mathrm{BC}$ criterion concerning gestational age 29 to $31+6 / 7$ weeks was not evidence-based, we wanted to determine whether the limited use of palivizumab affected the frequency of hospital visits among RSV-positive infants (termed "RSV-positive hospital visits"), comparing those who received approval for palivizumab and those who met the AAP/CPS criteria but did not receive approval for palivizumab.

\section{METHODS}

The study was conducted in Fraser Health, a BC health authority that encompasses 12 hospitals and 4 neonatal intensive care units (NICUs). Patients eligible for inclusion were infants with gestational age of 29 to $31+6 / 7$ weeks who were born at or transferred to any Fraser Health hospital between May 1, 2008, and April 30, 2011. The infants were identified through the Fraser Health perinatal database, which contains information collected from patient charts by health information management coders. The following data were obtained from the database: personal health number, date of birth, sex, date of discharge or transfer, location of transfer (if applicable), and gestational age. Infants were excluded if they had been transferred to a distant health authority, where a visit to a Fraser Health hospital or to the BC Children's Hospital would be unlikely. Infants without a personal health number were also excluded, because their data could not be matched across databases.

The dates of birth and discharge were reviewed to identify candidates for palivizumab according to the AAP/CPS guidelines, where infants less than 6 months of age at the start of the RSV season were candidates. Therefore, infants born after May 1 and discharged home before or during their first RSV season were considered candidates for palivizumab according to the AAP/CPS criteria. Infants who were still admitted to the NICU at the end of March of their first RSV season were not considered candidates, because in British Columbia the last dose of palivizumab is usually given in March. The RSV season in British 
Columbia is consistent from year to year, in terms of peak RSVrelated hospital visits.

All infants in any Fraser Health NICU were screened by either a nurse or a pharmacist using the provincial RSV application form. This form included information on birth date, gestational age, sex, birth weight, daycare attendance, siblings under 5 years, whether there were 5 or more people in the household, and whether there were 2 or more smokers in the household. The application form also allowed for highlighting of other high-risk indications, such as chronic lung disease. Once completed at the local site, the forms were forwarded to the provincial RSV centre, where the applications were approved or denied. For the purposes of the current study, the provincial palivizumab database was accessed to determine which Fraser Health infants with gestational age of 29 to $31+6 / 7$ weeks had been approved to receive palivizumab. During the study period, records of palivizumab administration were not consistently collected by the provincial centre, so it was not possible to confirm whether doses were actually administered. However, despite this inconsistent documentation, it would be very rare for a dose to be held or not administered in the Fraser Health authority for an infant born at 29 to $31+6 / 7$ weeks who had received approval for palivizumab.

The presence of RSV was determined with an immunoassay that detects the RSV antigen in respiratory specimens (Directigen chromatographic enzyme immunoassay, Becton Dickinson Microbiology systems). Infants less than 12 months of age who presented to either an emergency or inpatient ward at any Fraser Health hospital or to the BC Children's Hospital with symptoms of an upper or lower respiratory tract infection would usually have a nasopharyngeal wash to determine RSV status. Presentation to BC Children's Hospital was included for this aspect of the study because infants from the Fraser Health catchment area may be taken directly to the BC Children's Hospital. The laboratory records and the Fraser Health perinatal database were compared to identify infants born at 29 to $31+6 / 7$ weeks gestational age who also had an RSV-positive result. RSV-positive hospital visits during each year's RSV season (November through March) were specifically reviewed, because these admissions had the potential to be reduced with palivizumab use.

The date of an RSV-positive result was used to determine whether an infant was still admitted to the NICU or had a subsequent hospital visit following discharge from the NICU. In British Columbia, infants do not routinely receive palivizumab throughout their NICU stay; rather, the first dose is administered within days of the discharge.

Descriptive statistics were used to evaluate the study population. The frequency of RSV-positive hospital visits for infants who received approval for palivizumab and those who did not receive approval were compared using the Fisher exact test, with $p$ values less than 0.05 considered statistically significant.
Approval to conduct this study was obtained from the Fraser Health Research Ethics Board.

\section{RESULTS}

In this review, 406 infants were screened for eligibility, and 359 were included: 136 born in or transferred to a Fraser Health Hospital from May 2008 to April 2009, 114 from May 2009 to April 2010, and 109 from May 2010 to April 2011. Forty-six infants were excluded because they had been transferred to a distant health authority, and one infant was excluded because there was no personal health number.

Sixty-two of the infants did not meet the AAP/CPS criteria for palivizumab prophylaxis, because they had been born in April or remained in the NICU at the end of March. None of these infants had RSV-positive hospital visits. The remaining 297 infants would have been candidates for palivizumab according to the AAP/CPS guidelines (Figure 1). Of these, 46 (15.5\%) received approval for palivizumab under the $\mathrm{BC}$ criteria.

During the RSV season (November through March), 16 infants (4.5\% of the entire sample) had RSV-positive hospital visits. Of the 46 infants who received approval for palivizumab, 2 (4.3\%) had RSV-positive hospital visits, and of the 251 infants who met the AAP/CPS criteria but did not receive approval for palivizumab on the basis of the BC criteria, $14(5.6 \%)$ had RSV-positive hospital visits $(p>0.99)$.

Among the 16 infants with RSV-positive hospital visits, $8(50 \%)$ were male, and $8(50 \%)$ were born in November (Table 1). The dates of RSV-positive hospital visits followed an anticipated pattern, with the peak occurring in February.

Six (1.7\%) of the 359 infants had an RSV-positive result while admitted to the NICU; 2 of these had received approval for palivizumab, with no doses administered before the RSVpositive result. The RSV-positive result occurred at a mean age of 54 days (range 27 to 87 days). Ten (2.8\%) of the 359 infants, none of whom had received approval for palivizumab, had an RSV-positive result during a subsequent hospital visit. These RSV-positive results occurred at a mean age of 131 days (range 36 to 275 days).

\section{DISCUSSION}

Candidacy for RSV prophylaxis is often linked to pharmacoeconomics. The criteria used should be continually evaluated for efficacy and safety. In the current review of limited palivizumab use in British Columbia, the frequency of RSVpositive hospital visits during the RSV season was $4.5 \%$. The Canadian data from the IMpact-RSV Study Group showed that RSV-positive hospitalizations occurred in $14.7 \%$ and $8.8 \%$ of infants who received placebo and palivizumab, respectively. ${ }^{1}$ The Canadian population in the IMpact-RSV study had a high incidence of RSV-positive hospitalizations, more than twice the rates observed in the current study population, although that 


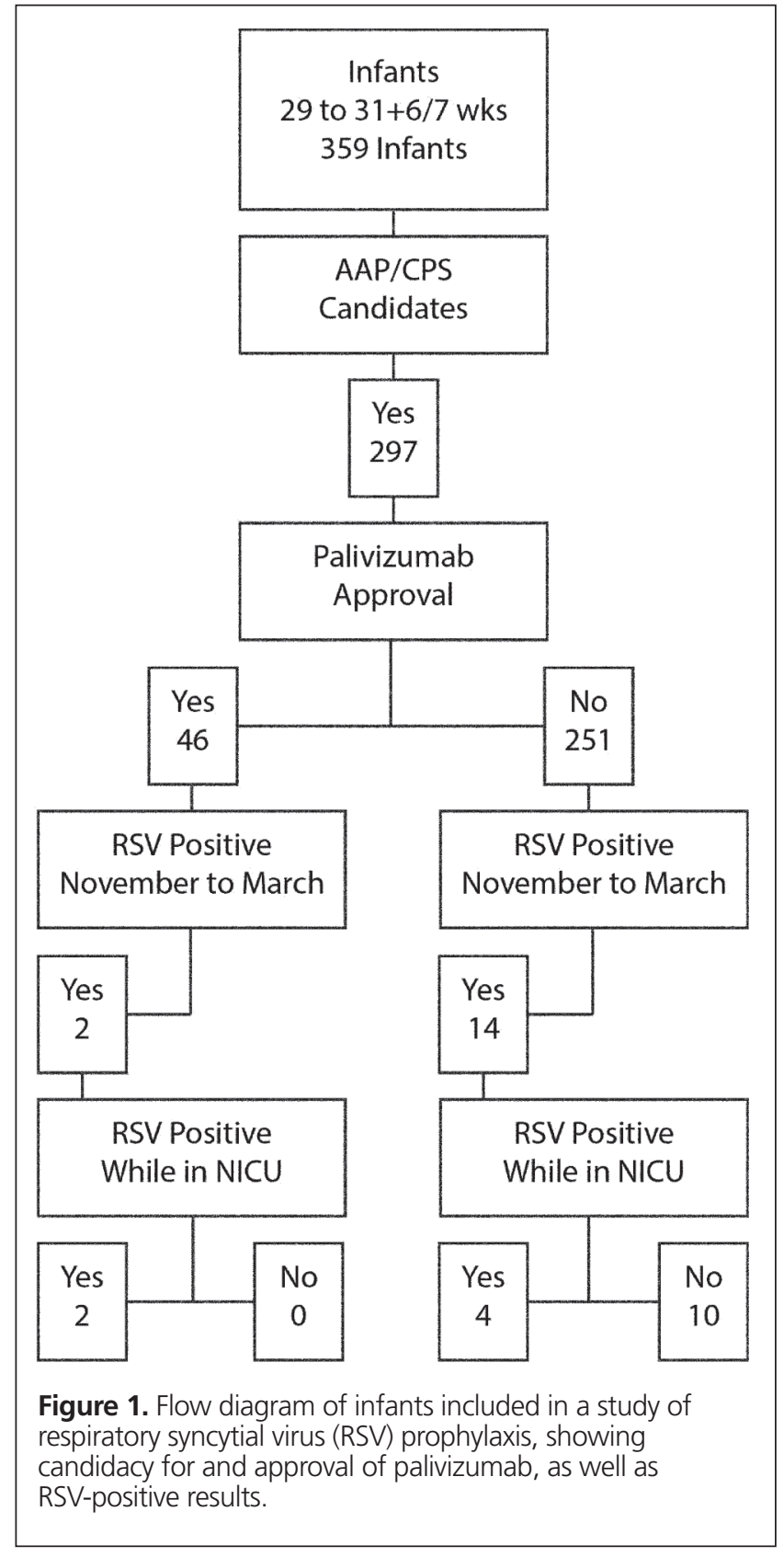

study included infants with a broader range of gestational ages. ${ }^{1}$ Among premature infants without bronchopulmonary disease, the IMpact-RSV Study Group found that $8.1 \%$ of infants who received placebo and $1.8 \%$ of those who received palivizumab had RSV-positive hospitalizations. ${ }^{1}$ Among infants of Englishor French-speaking parents or legal guardians with a gestational age of 29 to 32 weeks in the Canadian Registry of Palivizumab (CARESS), only $1.25 \%$ were hospitalized with RSV. ${ }^{8}$ Because Fraser Health cares for an ethnically diverse population, a significant proportion of families would have been excluded from the CARESS registry. ${ }^{8}$

There has been wide variation in the frequency of RSVpositive hospitalizations reported in the literature. In the general
Table 1. Characteristics of Infants with Hospital Visits Who Tested Positive for Respiratory Syncitial Virus (RSV)

\begin{tabular}{lrr} 
Characteristic & $\begin{array}{c}\text { No. (\%) of Infants } \\
(\boldsymbol{n}=\mathbf{1 6})\end{array}$ \\
\hline Sex & 8 & $(50)$ \\
Male & 8 & $(50)$ \\
Female & & \\
\hline Birth month & 8 & $(50)$ \\
November & 3 & $(19)$ \\
December & 1 & $(6)$ \\
February & 2 & $(12)$ \\
May & 1 & $(6)$ \\
July & 1 & $(6)$ \\
August & 3 & $(19)$ \\
\hline Month of positive RSV result & 4 & $(25)$ \\
December & 8 & $(50)$ \\
January & 1 & $(6)$ \\
February & & \\
March & 6 & $(38)$ \\
Location & 2 & $(12)$ \\
NICU & 7 & $(44)$ \\
Pediatric ICU & 1 & $(6)$ \\
Ward & &
\end{tabular}

population, the risk appears to be approximately $1 \%$ to $3 \%,{ }^{9,10}$ whereas the risk among premature infants is approximately $10 \%$ to $25 \% .{ }^{1,9,10}$ Use of palivizumab in premature infants has reduced the RSV-positive hospitalization rate to approximately $1.1 \%$ to $8.8 \% .^{1,8,10,11}$ The variation in hospitalization rates is likely due to the heterogeneity of the studies, including differences among patient populations, differing criteria for hospitalization, different definitions of RSV-positive hospitalization, and fluctuations in viral virulence. It is also possible that education about prevention of infection may influence the RSV-positive hospitalization rate. Such education is likely to be more consistent with enrolled study participants than the general population.

The variation in hospitalization rate makes it unclear what the target should be for infants involved in a prophylaxis program. If the goal of prophylaxis is to achieve a hospitalization rate for the highest-risk groups that is comparable with the rate for the lower-risk groups, then perhaps an RSV hospitalization rate of about $1 \%$ to $3 \%$ could be considered reasonable for high-risk premature infants, ${ }^{9,10}$ although preferably there would be no RSV-related hospitalizations at all. In the current study, $10(2.8 \%)$ infants who did not receive palivizumab had a subsequent hospital visit with RSV infection during RSV prophylaxis months. Of these infants, 2 were admitted to the intensive care unit, although the reason for admission and the significance of RSV-positive status were unknown. Notably, 6 (1.7\%) of the infants had an RSV-positive result while still admitted to the NICU. RSV is known to be transmitted in the hospital setting and can cause serious infection in these vulnerable infants. For the population studied here, it appears 
that additional precautions to limit RSV infection in the NICU are required. Administering palivizumab to candidates once they are stable in the NICU, instead of just before discharge, needs to be considered.

According to the $\mathrm{BC}$ criteria, infants who received approval for palivizumab required 3 or more risk factors in addition to prematurity, but the risk factors used were not validated for infants born at 29 to $31+6 / 7$ weeks gestational age before implementation of the criteria. Despite the additional requirements for palivizumab approval and the subsequent limited use of this agent, the frequency of RSV-positive hospital visits was not significantly different between infants who received approval and those who did not receive approval. Therefore, provision of palivizumab to those at higher risk according to the $\mathrm{BC}$ criteria may be adequate to keep RSV hospitalization rates similar to those for infants at lower risk. A risk factor-based approach has been validated among infants born at gestational age of 33 to 35 weeks. ${ }^{7}$ These older infants are reported to have RSV-positive hospitalization rates and complications similar to those of infants born at less than 33 weeks gestational age, so use of similar criteria may be appropriate. ${ }^{10-12}$ In the current review, 14 infants who did not receive approval for palivizumab had an RSV-positive hospital visit that might have been preventable. Further investigation of infants with RSV-positive results and their risk factors is required to determine whether there are any specific risk factors that could be used to inform future risk criteria.

This study had several limitations, including its retrospective nature and the assumed accuracy of the databases accessed to obtain study data. Infants with a false-negative RSV result and those who presented to a hospital in a different health authority may have been missed. The severity of RSV infection was also unknown. Documentation regarding palivizumab administration varied across the region, so it could not be confirmed whether infants with approval to receive palivizumab actually received it. However, our experience working in the largest RSV clinic in Fraser Health indicates that nearly all doses are administered as expected, with only rare situations where a dose is not given.

In the time since these data were collected, debate has continued about which patients should receive palivizumab. The consensus conference statement of Pignotti and others, ${ }^{13}$ which encompassed expert opinion and available evidence, suggests that palivizumab prophylaxis is not recommended for infants of gestational age 29 weeks or more unless there is a comorbid condition. Yet the review by Homaira and others ${ }^{14}$ recommended the use of palivizumab for infants born at less than 33 weeks gestational age. There is also a large variation in the efficacy of palivizumab, depending on the patient group being reviewed. Wegzyn and others ${ }^{15}$ reported a 39\% to $82 \%$ relative reduction in RSV-related hospitalizations for palivizumab compared with placebo.

The limited evidence for clear clinical benefit of palivizumab prophylaxis for all premature infants has led to revisions in the guidelines of the AAP and the CPS. ${ }^{16,17}$ The AAP guidelines suggest that among infants born after 29 weeks gestational age, only those with chronic lung disease require prophylaxis with palivizumab. ${ }^{16}$ The current CPS guidelines state that it is reasonable, but not essential, to offer palivizumab to infants born at less than 30 weeks gestational age, and that infants over 30 weeks gestational age without chronic lung disease should not be given palivizumab. ${ }^{17}$

Although the AAP/CPS guidelines are restrictive, many BC infants born at 29 to $31+6 / 7$ weeks gestational age continue to receive palivizumab. The BC criteria for 2016 still limited prophylaxis for infants born at gestational age 29 to $31+6 / 7$ weeks, although the qualifications based on risk factors have been modified. To qualify now, the infants must be discharged home after October 1 and must reach a minimum risk factor score. ${ }^{18}$ In contrast, the 2016 palivizumab prophylaxis programs in some other provinces continued to include infants who were born at less than 32 weeks gestational age and who were less than 6 months of age at the start of or during the local RSV season. ${ }^{19-21}$ There appears to be an ongoing need to identify which patients will benefit the most from palivizumab prophylaxis. The information in the current review may help to inform future guidelines, although more research into optimal prophylaxis strategies in clinical practice is required.

\section{CONCLUSION}

Despite the additional requirements for palivizumab approval in British Columbia that were in effect at the time of our review, the frequency of RSV-positive hospital visits was not significantly different for infants with and without palivizumab approval. During the RSV season, the limited use of palivizumab in this study population resulted in an overall frequency of $4.5 \%$ for RSV-positive hospital visits, consistent with other studies, which have reported rates of $1.1 \%$ to $8.8 \%$ among premature infants who received palivizumab. ${ }^{1,8,10,11}$ When RSV-positive infants in the NICU were excluded, only $2.8 \%$ of the infants in this study had RSV-positive hospital visits. Therefore, a risk factor-based approach for RSV prophylaxis, limiting the use of palivizumab in infants born at 29 to $31+6 / 7$ weeks, led to reasonable rates for RSV-positive hospital visits in this population. Use of these criteria requires validation with a larger prospective study.

\section{References}

1. IMpact-RSV Study Group. Palivizumab, a humanized respiratory syncytial virus monoclonal antibody, reduces hospitalization from respiratory syncytial virus infection in high-risk infants. Pediatrics. 1998;102(3 Pt 1):531-7.

2. Robinson JL; Canadian Paediatric Society, Infectious Diseases and Immunization Committee. Preventing respiratory syncytial virus infections. Paediatr Child Health. 2011;16(8):487-8.

3. American Academy of Pediatrics, Committee on Infectious Diseases. Modified recommendations for use of palivizumab for prevention of respiratory syncytial virus infections. Pediatrics. 2009;124(6):1694-701.

4. Fitzgerald DA. Preventing RSV bronchiolitis in vulnerable infants: the role of palivizumab. Paediatr Respir Rev. 2009;10(3):143-7. 
5. Mori M, Kawashima H, Nakamura H, Nakagawa M, Kusuda S, Saji T, et al.; Surveillance Committee for Severe RSV Infection. Nationwide survey of severe respiratory syncytial virus infection in children who do not meet indications for palivizumab in Japan. J Infect Chemother. 2011;17(2):254-63.

6. Simon A, Nowak H, Sterz R. Use of palivizumab in Germany: data from 20022007. Klin Padiatr. 2011;223(5):292-8.

7. Paes B, Steele S, Janes M, Pinelli J. Risk-scoring tool for respiratory syncytial virus prophylaxis in premature infants born at 33-35 completed weeks' gestational age in Canada. Curr Med Res Opin. 2009;25(7):1585-91.

8. Mitchell I, Paes BA, Li A, Lanctôt KL; CARESS investigators. CARESS: the Canadian registry of palivizumab. Pediatr Infect Dis J. 2011;30(8):651-5.

9. Paes BA, Mitchell I, Banerji A, Lanctôt K, Langley JM. A decade of respiratory syncytial virus epidemiology and prophylaxis: translating evidence into everyday clinical practice. Can Respir J. 2011;18(2):e10-9.

10. Lacaze-Masmonteil T, Rozé JC, Fauroux B; French Pediatricians' Group of Synagis Patients' Name-Based Programs. Incidence of respiratory syncytial virus-related hospitalizations in high-risk children: follow-up of a national cohort of infants treated with palivizumab as RSV prophylaxis. Pediatr Pulmonol. 2002;34(3):181-8.

11. Frogel M, Nerwen C, Cohen A, VanVeldhuisen P, Harrington M, Boron M; Palivizumab Outcomes Registry Group. Prevention of hospitalization due to respiratory syncytial virus: results from the Palivizumab Outcomes Registry. J Perinatol. 2008;28(7):511-7.

12. Horn SD, Smout RJ. Effect of prematurity on respiratory syncytial virus hospital resource use and outcomes. J Pediatr. 2003;143(5 Suppl):S133-41.

13. Pignotti MS, Carmela LM, Pugi A, De Masi S, Biermann KP, Galli L, et al. Consensus conference on the appropriateness of palivizumab prophylaxis in respiratory syncytial virus disease. Pediatr Pulmonol. 2016;51(10):1088-96.

14. Homaira N, Rawlinson W, Snelling TL, Jaffe A. Effectiveness of palivizumab in preventing RSV hospitalization in high risk children: a real-world perspective. Int J Pediatr. 2014;2014:571609.

15. Wegzyn C, Toh LK, Notario G, Biguenet S, Unnebrink K, Park C, et al. Safety and effectiveness of palivizumab in children at high risk of serious disease due to respiratory syncytial virus infection: a systematic review. Infect Dis Ther. 2014;3(2):133-58.

16. American Academy of Pediatrics, Committee on Infectious Diseases; American Academy of Pediatrics, Bronchiolitis Guidelines Committee. Updated guidance for palivizumab prophylaxis among infants and young children at increased risk of hospitalization for respiratory syncytial virus infection. Pediatrics. 2014;134(2):415-20.

17. Robinson JL, Le Saux N; Canadian Paediatric Society, Infectious Diseases and Immunization Committee. Preventing hospitalizations for respiratory syncytial virus infection. Paediatr Child Health. 2015;20(6):321-6. Also available from: www.cps.ca/en/documents/position/preventing-hospitalizations-for-rsvinfections [updated 2016 May 12].
18. BC RSV immunoprophylaxis program: administrative manual and decision support tool. Vancouver (BC): Provincial Health Services Authority; 2016 [cited 2017 Jan 3]. Available from: www.childhealthbc.ca/sites/default/files/ RSV\%20Manual\%20\%26\%20DST\%20-\%20201617.pdf

19. Alberta respiratory syncytial virus (RSV) prevention program: information for health professionals. Alberta Health Services; 2016 [cited 2016 Oct 7]. Available from: www.albertahealthservices.ca/info/rsvprogram.aspx

20. Publicly funded drug programs: Respiratory syncytial virus prophylaxis for high-risk infants program. Toronto $(\mathrm{ON})$ : Ontario Ministry of Health and Long-Term Care; 2016 [cited 2016 Oct 7]. Available from: www.health. gov.on.ca/en/pro/programs/drugs/funded_drug/fund_respiratory.aspx

21. Respiratory syncytial virus prophylaxis program: Saskatchewan protocol 2016-2017 season. Saskatoon (SK): Saskatchewan RSV Prophylaxis Program; 2016 [cited 2016 Oct 7]. Available from: https://www.saskatoon healthregion.ca/locations_services/Services/Childrens-Services/RSVProgram/Documents/Protocol\%20for\%20SK\%20\%202016-2017-FINAL.pdf

Brandi Newby, BSCPharm, ACPR, is Coordinator, Clinical and Distribution Pharmacy Services, Neonatal and Pediatric Pharmacy, Surrey Memorial Hospital, Surrey, British Columbia.

Todd Sorokan, MD, FRCPC, is Staff Neonatologist with the Department of Neonatology, Surrey Memorial Hospital, Surrey, British Columbia. He is also Clinical Assistant Professor with the University of British Columbia, Vancouver, British Columbia.

Competing interests: Both authors represented the Fraser Health authority on the BC Respiratory Syncytial Virus (RSV) Immunoprophylaxis Task Force; they were not involved in selecting the criteria for RSV prophylaxis. No financial competing interests were declared.

\section{Address correspondence to:}

Brandi Newby

Neonatal and Pediatric Pharmacy, Room 2-602

Critical Care Tower

Surrey Memorial Hospital

13750 96th Avenue

Surrey BC V3V 1 Z2

e-mail: Brandi.newby@fraserhealth.ca

Funding: None received. 